hep-th/0303068

FIT HE - 03-01

Kagoshima HE-03-1

\title{
Newton's law in de Sitter brane
}

\author{
Kazuo Ghoroku ${ }^{1}$ \\ Fukuoka Institute of Technology, Wajiro, Higashi-ku \\ Fukuoka 811-0295, Japan \\ Akihiro Nakamura ${ }^{2}$ \\ Department of Physics, Kagoshima University, Korimoto 1-21-35 \\ Kagoshima 890-0065, Japan \\ Masanobu Yahiro ${ }^{3}$ \\ Department of Physics and Earth Sciences, University of the Ryukyus, \\ Nishihara-chou, Okinawa 903-0213, Japan
}

\begin{abstract}
Newton potential has been evaluated for the case of dS brane embedded in Minkowski, $\mathrm{dS}_{5}$ and $\mathrm{AdS}_{5}$ bulks. We point out that only the $\mathrm{AdS}_{5}$ bulk might be consistent with the Newton's law from the brane-world viewpoint when we respect a small cosmological constant observed at present universe.
\end{abstract}

\footnotetext{
${ }^{1}$ gouroku@dontaku.fit.ac.jp

${ }^{2}$ nakamura@sci.kagoshima-u.ac.jp

3 yahiro@sci.u-ryukyu.ac.jp
} 


\section{Introduction}

It is quite expectable to consider our four dimensional world as a brane like the one proposed in 1, 2. The recent interest is the de Sitter (dS) brane due to the observation of a small but finite cosmological constant in the present universe. The $4 \mathrm{~d}$ Newton's law is guaranteed also on dS brane by the confirmation of the localization of graviton on this brane for wide range of bulk configurations, for example $\mathrm{AdS}_{5}$ and $\mathrm{dS}_{5}[3$, 4] as in 2]. The non-localized modes, which are called as Kaluza-Klein (KK) modes, give corrections to the Newton potential. They are dependent on the configuration of the bulk space. For the Randall-Sundrum (RS) brane, massive KK modes yields correction like $1 / r^{3}$ [2, 5, 6], and it is nicely understood from an idea of AdS/CFT correspondence [6. After that, corrections to the Newton's law on the brane in the other bulk have been studied [7, 8, 9, 10, 12, 13]. However some points have not yet been made clear.

It is then of interest and importance to make more analysis about what kind of corrections appear. Our purpose is to see the corrections coming from KK modes to Newton's law in the case of $\mathrm{dS}$ brane for $5 \mathrm{~d}$ Minkowski, $\mathrm{AdS}_{5}$ and $\mathrm{dS}_{5}$ bulks. In Section 2, we give our model and the $5 \mathrm{~d}$ graviton propagator to study the $4 \mathrm{~d}$ Newton potential. In Section 3, the gravitational potentials are examined under a reasonable setting. Summary is given in the final section.

\section{Graviton propagator}

The five-dimensional gravitational action is obtained, in the Einstein frame, as ${ }^{4}$

$$
S=\frac{1}{2 \kappa^{2}}\left\{\int d^{5} X \sqrt{-G}\left(R-2 \Lambda+L_{m}\right)+2 \int d^{4} x \sqrt{-g} K\right\}-\tau \int d^{4} x \sqrt{-g}
$$

where $1 / 2 \kappa^{2}=M^{3}$ and $K$ denotes the extrinsic curvature on the boundary. Five and four dimensional metrics are denoted as $G_{M N}$ and $g_{\mu \nu}$. The Lagrangian density $L_{m}$ represents a contribution from matter, and not needed to construct a background metric. The last term shows a brane action. The Einstein equation derived from $S$ is solved under an assumption,

$$
d s^{2}=A^{2}(y)\left\{-d t^{2}+a_{0}^{2}(t) \gamma_{i j}\left(x^{i}\right) d x^{i} d x^{j}\right\}+d y^{2},
$$

where coordinates parallel and transverse to a brane are denoted by $x^{\mu}=\left(t, x^{i}\right)$ and $y$, respectively. The brane is located at $y=0$. We restrict our interest here to the case of a Friedmann-Robertson-Walker type (FRW) universe. Then, the three-dimensional metric $\gamma_{i j}$ is described in Cartesian coordinates as $\gamma_{i j}=\left(1+k \delta_{m n} x^{m} x^{n} / 4\right)^{-2} \delta_{i j}$, where the parameter values $k=0,1,-1$ correspond to flat, closed, and open universe, respectively. The scale factors, $a_{0}(t)$ and $A(y)$, are obtainable from the Einstein equation [3].

\footnotetext{
${ }^{4}$ Definitions taken here are, $R_{\nu \lambda \sigma}^{\mu}=\partial_{\lambda} \Gamma_{\nu \sigma}^{\mu}-\cdots, R_{\nu \sigma}=R_{\nu \mu \sigma}^{\mu}$ and $\eta_{A B}=\operatorname{diag}(-1,1,1,1,1)$. Five dimensional suffices are denoted by capital Latin and four dimensional ones by Greek letters.
} 
A perturbed metric $h_{i j}$, representing graviton, is assumed to have a form

$$
d s^{2}=A^{2}(y)\left\{-d t^{2}+a_{0}(t)^{2}\left[\delta_{i j}+h_{i j}\left(t, x^{i}, y\right)\right] d x^{i} d x^{j}\right\}+d y^{2},
$$

where the case $k=0$ is taken. In this case, $a(t)=e^{\sqrt{\lambda t}}$ and $\lambda$ is the $4 \mathrm{~d}$ cosmological constant, which is written by other parameters as [3]

$$
\lambda=\kappa^{4} \tau^{2} / 36+\Lambda / 6 .
$$

A traceless and transverse component, $h$, of the perturbation is relevant to Newton's law on our brane and its corrections. Projecting the component out with conditions $h_{i}^{i}=0$ and $\nabla_{i} h^{i j}=0$, its $5 \mathrm{~d}$ propagator, $\Delta_{5}$, should satisfy the following equation,

$$
\square_{5} \Delta_{5}\left(x, y ; x^{\prime}, y^{\prime}\right)=\frac{\delta^{4}\left(x-x^{\prime}\right) \delta\left(y-y^{\prime}\right)}{\sqrt{-G}},
$$

where

$$
\square_{5} \equiv \frac{1}{\sqrt{-G}} \partial_{N} \sqrt{-G} G^{N L} \partial_{L}=\frac{1}{A^{2}(y)} \square_{4}+\left(\partial_{y}^{2}+\frac{4}{A}\left(\partial_{y} A\right) \partial_{y}\right)
$$

and $\square_{4}=-\partial_{t}^{2}-3 \dot{a}_{0} / a_{0} \partial_{t}+\partial_{i}^{2} / a_{0}^{2}$.

When a new coordinate $z$ and a redefined propagator $\Delta\left(x, z ; x^{\prime}, z^{\prime}\right)$ are introduced as $\partial z / \partial y= \pm A^{-1}$ and $\Delta_{5}=A(z)^{-3 / 2} \Delta A\left(z^{\prime}\right)^{-3 / 2}, \Delta$ is solved as

$$
\begin{gathered}
\Delta\left(x, z ; x^{\prime}, z^{\prime}\right)=u(0, z) \Delta_{0}\left(x, x^{\prime}\right) u\left(0, z^{\prime}\right)+\int_{m_{0}^{2}}^{\infty} d m^{2} u(m, z) \Delta_{m}\left(x, x^{\prime}\right) u\left(m, z^{\prime}\right), \\
{\left[-\partial_{z}^{2}+V(z)\right] u(m, z)=m^{2} u(m, z),} \\
\left(\square_{4}^{2}-m^{2}\right) \Delta_{m}=\frac{\delta^{4}\left(x-x^{\prime}\right)}{\sqrt{-g}},
\end{gathered}
$$

where $V(z)=\frac{9}{4}\left(\partial_{y} A\right)^{2}+\frac{3}{2} A \partial_{y}^{2} A$, and $m$ corresponds the mass observed on the brane, as seen in Eq. (9). The explicit form of the $4 \mathrm{~d}$ propagator, $\Delta_{m}$, on AdS brane is not expressed here since we don't use it. The eigenmodes, the solutions of (8), consist of a zero mode $u(0, z)$ and continuum KK modes $u(m, z)$ with $m^{2}>m_{0}^{2}$, for the given bulks, where $m_{0}^{2}=9 \lambda / 4[3]$. The normalization of $u(0, z)$ is given by demanding

$$
\int_{z_{0}}^{\infty} d z u(0, z)^{2}=1
$$

This integration is easily performed numerically. As for the KK mode $u(m, z)$, its normalization is obtained by imposing the following condition

$$
\int_{z_{0}}^{\infty} d z u(m, z) u\left(m^{\prime}, z\right)=\delta\left(m^{2}-m^{\prime 2}\right) .
$$


The explicit form of $u(m, z)$ can be obtained in terms of the two independent and complex-conjugate solutions (denoted by $F_{1}$ and $F_{2}$ below) of the equation (8), together with the boundary condition on a brane [3],

$$
u^{\prime}\left(z_{0}\right)=-\frac{\kappa^{2} \tau}{4} u\left(z_{0}\right)
$$

The result is summarized as

$$
\begin{gathered}
u(m, z)=\frac{1}{2 i} \sqrt{\frac{1}{\pi \alpha}}\left[e^{i \delta_{0}(\alpha)} F_{1}(z)-e^{-i \delta_{0}(\alpha)} F_{2}(z)\right], \\
e^{2 i \delta_{0}(\alpha)}=\frac{F_{2}^{\prime}\left(z_{0}\right)+\frac{\kappa^{2} \tau}{4} F_{2}\left(z_{0}\right)}{F_{1}^{\prime}\left(z_{0}\right)+\frac{\kappa^{2} \tau}{4} F_{1}\left(z_{0}\right)},
\end{gathered}
$$

where $^{\prime}=\partial / \partial_{z}$ and

$$
\begin{gathered}
F_{1}(z)=Y_{2}^{-i d} F_{1}\left(b_{1}, b_{2} ; b_{3} ;-Y\right), \quad F_{2}(z)=Y_{2}^{i d} F_{1}\left(b_{1}^{\prime}, b_{2}^{\prime} ; b_{3}^{\prime} ;-Y\right) \quad \text { for } \Lambda<0, \\
F_{1}(z)=X_{2}^{-i d} F_{1}\left(b_{1}, b_{2} ; b_{3} ; X\right), \quad F_{2}(z)=X_{2}^{i d} F_{1}\left(b_{1}^{\prime}, b_{2}^{\prime} ; b_{3}^{\prime} ; X\right) \quad \text { for } \Lambda>0, \\
Y=\frac{1}{\sinh ^{2}(\sqrt{\lambda} \mathrm{z})}, \quad X=\frac{1}{\cosh ^{2}(\sqrt{\lambda} \mathrm{z})}, \quad d=\frac{\sqrt{-9+4 m^{2} / \lambda}}{4}, \\
b_{1}=-\frac{3}{4}-i d, \quad b_{2}=\frac{5}{4}-i d, \quad b_{3}=1-2 i d, \\
b_{1}^{\prime}=-\frac{3}{4}+i d, \quad b_{2}^{\prime}=\frac{5}{4}+i d, \quad b_{3}^{\prime}=1+2 i d .
\end{gathered}
$$

Here ${ }_{2} F_{1}\left(b_{1}, b_{2} ; b_{3} ; X\right)$ denotes the Gauss's hypergeometric function. For $m>m_{0}, F_{1}(z)$ represents an outgoing wave asymptotically, while $F_{2}(z)$ does an incoming wave, and both are complex conjugate to each other. To see that this solution satisfies the above normalization condition, it is convenient to use the followings two relations. The first one is the following asymptotic form at large $z$,

$$
u(m, z) \rightarrow \sqrt{\frac{1}{\pi \alpha}} \sin \left(\alpha z+\delta_{\alpha}\right),
$$

where $\alpha=\sqrt{m^{2}-m_{0}^{2}}, \delta_{\alpha}$ means a phase dependent on $\alpha$. The second relation is given by using (18) as,

$$
u(m, z) u\left(m^{\prime}, z\right)=\frac{1}{m^{2}-m^{\prime 2}}\left\{u(m, z) \partial_{z}^{2} u\left(m^{\prime}, z\right)-u\left(m^{\prime}, z\right) \partial_{z}^{2} u(m, z)\right\} .
$$

The present universe implies a small $\lambda$, then we concentrate our discussion on such a case. An observational time $t$ of Newton's law is much smaller than the cosmic age, $1 / \sqrt{\lambda} \sim 10$ Gyr. For the case of such a small time, the scale factor $a_{0}=\exp \sqrt{\lambda} t$ on a brane is well approximated by $a_{0}=1$, and the de-Sitter propagator denoted above by 
$\Delta_{m}$ can be approximated into the one in Minkowski space. The approximate form of the $5 \mathrm{~d}$ propagator, valid at $\sqrt{\lambda}\left|t-t^{\prime}\right| \ll 1$, is then obtained as

$$
\begin{aligned}
\Delta\left(x, z ; x^{\prime}, z^{\prime}\right)= & u(0, z) u\left(0, z^{\prime}\right) \int \frac{d^{4} p}{(2 \pi)^{4}} \frac{e^{i p\left(x-x^{\prime}\right)}}{-p^{2}+i \epsilon} \\
& +\int_{m_{0}^{2}}^{\infty} d m^{2} u(m, z) u\left(m, z^{\prime}\right) \int \frac{d^{4} p}{(2 \pi)^{4}} \frac{e^{i p\left(x-x^{\prime}\right)}}{-p^{2}-m^{2}+i \epsilon} .
\end{aligned}
$$

In the limit of $\lambda=0$, Eq. (22) is correct.

\section{Corrections to Newton's Law}

The static potential $\tilde{U}(r)$ between two objects of unit mass on a brane is defined as [5]

$$
\begin{aligned}
U(r) & =\tilde{U}(r) / \kappa^{2}=-\left.\int_{-\infty}^{\infty} d t \Delta_{5}\left(t, x_{i}, y ; t^{\prime}, x_{i}^{\prime}, y^{\prime}\right)\right|_{y=y^{\prime}=0, t^{\prime}=0} \\
& =-\left.\int_{-\infty}^{\infty} d t \Delta\left(t, x_{i}, z ; t^{\prime}, x_{i}^{\prime}, z^{\prime}\right)\right|_{z=z^{\prime}=z_{0}, t^{\prime}=0},
\end{aligned}
$$

where $r=\left|\vec{x}-\overrightarrow{x^{\prime}}\right|$. Inserting the approximate form (22) into Eq. (23) leads to $U(r)=U_{0}+\Delta U$ for

$$
U_{0} \equiv \frac{u\left(0, z_{0}\right)^{2}}{4 \pi r}, \quad \Delta U \equiv \int_{m_{0}^{2}}^{\infty} d m^{2} u\left(m, z_{0}\right)^{2} \frac{e^{-m r}}{4 \pi r} .
$$

The term $U_{0}$ guarantees Newton's law, and $\Delta U$ represents its correction. The correction depends on the magnitude of KK mode $u\left(m, z_{0}\right)$ on a brane.

Particularly at $z=z_{0}$, namely on a brane, the KK mode has a simple form

$$
u\left(m, z_{0}\right)=-\sqrt{\frac{1}{\pi \alpha}} \frac{\alpha}{\left|F_{1}^{\prime}\left(z_{0}\right)+\frac{\kappa^{2} \tau}{4} F_{1}\left(z_{0}\right)\right|},
$$

where use has been made of $F_{1}^{\prime}(z) F_{2}(z)-F_{2}^{\prime}(z) F_{1}(z)=2 i \alpha$. Inserting Eq. (25) into Eq. (24) leads to

$$
\Delta U=\frac{1}{2 \pi^{2} r} \int_{m_{0}}^{\infty} d m \frac{m \alpha}{F} e^{-m r}, \quad F \equiv\left|F_{1}^{\prime}\left(z_{0}\right)+\frac{\kappa^{2} \tau}{4} F_{1}\left(z_{0}\right)\right|^{2} .
$$

Equation (26) thus obtained is based on Eq. (22) which is valid for $\sqrt{\lambda}\left|t-t^{\prime}\right| \ll 1$. So Eq. (26) is accurate for $\sqrt{\lambda} r \ll 1$, because the distance $r$ between two massive objects is related to the propagation time of graviton $\left|t-t^{\prime}\right|$ as $r \approx\left|t-t^{\prime}\right|$. Particularly in the limit $\lambda \rightarrow 0$, Eq. (26) is correct for any $r$.

The correction (26) is different from the corresponding one in Ref. [10], since the normalization and boundary conditions, (11) and (12), are not imposed there [11. Then their results could not reproduce the $1 / r^{3}$ correction in the limit of $\lambda \rightarrow 0$. In our case, it can be seen as shown below. 


\subsection{Randall-Sundrum brane}

As for the Randall-Sundrum brane, in which $\lambda=0$, corrections to Newton's law are well known at $r \gg L$ [2], where $L$ is the radius defined by $L=\sqrt{6 /|\Lambda|}$. In this subsection, the corrections are analyzed for both regions of $r<L$ and $r>L$.

For $\lambda=0$, the corresponding solutions $F_{1}(z)$ and $F_{2}(z)$ of the equation (88) are given as $F_{1}(z)=\sqrt{\pi m z / 2} H_{2}^{(1)}(m z)$ and $F_{2}(z)=\sqrt{\pi m z / 2} H_{2}^{(2)}(m z)$, where $H_{n}^{(1,2)}(x)=$ $J_{n}(x) \pm i N_{n}(x)$ for the Bessel functions $J_{n}(x)$ and $N_{n}(x)$ of integer $n$. Since $z_{0}=L$ in the case, $F$ in Eq. (26) is expressed as $F=\pi m^{3} L\left|H_{1}^{(1)}(m L)\right|^{2} / 2$, indicating that $F \rightarrow 2 m /(\pi L)$ at the small limit of $m L$ and $F \rightarrow m^{2}$ at the large limit.

Firstly, consider the region $r \gg L$ where $F$ is approximated by the one for small $m L$ since $m$ and $r$ are mutually conjugate due to the factor $e^{-m r}$ in the integrand in (26). Then we obtain

$$
\Delta U \sim L /\left(4 \pi r^{3}\right),
$$

which leads to a well-known result $\Delta U / U_{0}=L^{2} /\left(2 r^{2}\right)[6$.

While in the region of small $r, L \gg r$, the potential can be estimated by the approximation of $F \sim m^{2}$, and we obtain

$$
\Delta U \sim 1 /\left(2 \pi^{2} r^{2}\right) .
$$

It indicates $\Delta U / U_{0}=L /(\pi r) \gg 1$, then the pole contribution of $1 / r$ is small and $5 \mathrm{~d}$ Newton's law appears as the dominant potential in the region $r \ll L$ as expected. This is pointed out also in [7, 8, 9, 12,

\section{2 dS brane with small $\lambda$}

The dS brane, in which $\lambda>0$, can be embedded in three types of bulks, $\mathrm{AdS}_{5}$ [14, 15], $\mathrm{dS}_{5}$ [15] and the $5 \mathrm{~d}$ Minkowski space [16]. In this case, two scale parameters appear in studying the potential at some region of $r$. Due to the relation (44), the region of $r$ in the two bulks, $\mathrm{dS}_{5}$ and the $5 \mathrm{~d}$ Minkowski space, is restricted to a short range region as shown below.

For the $5 \mathrm{~d}$ Minkowski space as a simple case, $F_{1}$ is obtained as $F_{1}=\exp (i \alpha z)$, leading to $F=m^{2}$. This is understood also from the above approximate form of $F$ at large $m L$. For 5 d Minkowski, $L=\infty$ and this implies that the potential in this case expresses the exact $5 \mathrm{~d}$ limit at any $r$.

The similar situation is seen also in the dS bulk. Consider it with the radius $L=\sqrt{6 / \Lambda}$. As noted above in (4i), $\lambda$ is related to $\Lambda$ as $\lambda=\kappa^{4} \tau^{2} / 36+\Lambda / 6$. This relation shows that $\lambda>\Lambda / 6$ for the dS bulk, that is, $1 \leq m / m_{0}<m L$. Then we can not see the region of $L \ll r$ or $m L \ll 1$. Therefore, $1 / r^{3}$ correction can not be seen in this case. And the available region is restricted to the short range region. As a result, it is easy to see $5 \mathrm{~d}$ potential, $1 / r^{2}$, at small $r$ as in the case of RS given above. 
Further, we can see that the potential $1 / r$ coming from the trapped zero mode is not the leading term any more in this region. The $1 / r$ term $U_{0}$ depends on the magnitude $u\left(0, z_{0}\right)$ on the brane. The magnitude is estimated by using the explicit form of the solutions given above (16). By setting as $m=0$ we obtain, $u\left(0, z_{0}\right)=$ $d_{1} F_{1}\left(z_{0}\right)$ with $F_{1}(z)=(\cosh \sqrt{\lambda} z)^{-3 / 2}$ and $d_{1}=\left\langle F_{1} \mid F_{1}\right\rangle_{z}^{-1 / 2}$. It is impossible to integrate $F_{1}^{2}$ over $z$ analytically. So an order estimate is made for $d_{1}$ with the relation, $\{\exp (\sqrt{\lambda} z)\}^{-3}<F_{1}^{2}<\{\exp (\sqrt{\lambda} z) / 2\}^{-3}$, which is valid for any positive $z$. Making an analytic integration for each function in the lower and upper bounds of the relation, one can obtain the relation $\sqrt{m_{0} / 4} f^{3 / 2}<u\left(0, z_{0}\right)<\sqrt{2 m_{0}} f^{3 / 2}$, where $f(\beta)=1+\sqrt{1-\beta^{2}}$ for $\beta \equiv \sqrt{\Lambda / 6 \lambda}=3 /\left(2 L m_{0}\right)$. This indicates that $u\left(0, z_{0}\right)$ is of order $\sqrt{m_{0}}$, because $1<f<2$ in the entire region $0<\beta<1$. This order estimation leads to $U_{0} \approx m_{0} /(4 \pi r)$ for the case of dS bulk.

The terms $U_{0}$ and $\Delta U$ calculated above lead to $\Delta U / U_{0} \approx 1 /\left(r m_{0}\right) \gg 1$ for $r \ll 1 / m_{0}$. From the observation or our assumption $\lambda \sim m_{0}^{2} \ll M^{2}$, this estimation is justified. This indicates that the present universe is not embedded in the $\mathrm{dS}$ or Minkowski bulk when we consider according to our brane model.

As for $\mathrm{AdS}_{5}$, (41) gives no considerable constraint on $m L$ or the range of $r$. As a matter of fact, the situation is similar to the case of the RS brane since $m_{0}$ is so small compared to the value of $1 / L$. However $F$ is slightly different from the one of RS, especially near $m=m_{0}$. While the function $F$ has the same form in the large limit of $m^{2} /|\Lambda|$, since $F_{1}$ tends to $\exp (i \alpha z)$ in the limit. Figure 1 shows the behavior of $F$ at smaller $m$ for various $\lambda$. The value of $\lambda$ can be measured by $\Lambda$ in the theory, so we take as $10^{-3.1}<\lambda /|\Lambda|<10^{-4.6}$ in the figure. But it should be taken at about $\sim 10^{-15}$ actually, then the realistic case is infinitely near the RS limit.

Generally, it is possible to approximate $F$ by

$$
F \approx c_{0}+c_{1} m+c_{2} m^{2}
$$

where $c_{i}$ are dependent on $\mu=\sqrt{-\Lambda / 6}$ and $\lambda$. When $\lambda$ becomes small, $F$ approaches to the one of RS brane, since $c_{0} \rightarrow 0, c_{1} \rightarrow 2 / \pi L, c_{2} \rightarrow 1$ in the limit. So it would be possible to find a similar potential at large $r$ to the one of RS case when the parameters $\lambda$ and $\Lambda$ are appropriately chosen.

However the essential difference from the RS case would be seen in the ratio $\Delta U / U_{0}$, which represents the ratio of the correction like $1 / r^{3}$ and the leading term of $1 / r$. It would be expected that this ratio shifts from the RS case, $\Delta U / U_{0}=L^{2} /\left(2 r^{2}\right)$, and can be written as

$$
\Delta U / U_{0}=f(L, \lambda) /\left(2 r^{2}\right) .
$$

To study the meaning of this difference is an interesting problem from the theoretical viewpoint of AdS/CFT correspondence. We will discuss this issue in the future paper.

As a result of this section, we can say that the favorable bulk configuration of the brane-world would be the $\mathrm{AdS}_{5}$ 


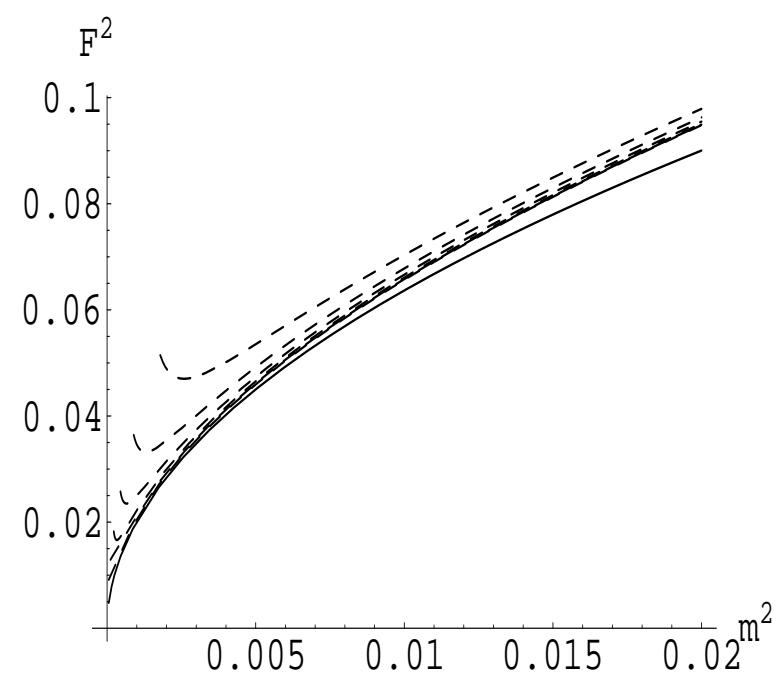

Fig. 1: The solid curve shows $\frac{2}{\pi} m$, and the dotted curves represent $F$ for $\mu=1$ and $\lambda=10^{-2.8-0.3 N}$ where $N=1 \sim 6$ from the highest to the lowest one. The end points at small $m$ of the dotted curves correspond to the values at $m=m_{0}$ for each $\lambda$. We can see that $F$ approaches to the RS limit as $\lambda \rightarrow 0$.

\section{Summary}

In this paper, Newton potential has been evaluated for the case of dS brane embedded in Minkowski, $\mathrm{dS}_{5}$ and $\mathrm{AdS}_{5}$ bulks.

For this purpose, an approximate propagator (22) has been derived, which is valid at $\sqrt{\lambda}\left|t-t^{\prime}\right| \ll 1$. Then on the basis of the propagator, the static potential $U(r)$ has been divided into $U_{0}$ which guarantees Newton's law and $\Delta U$ which represents its correction. The formula (26), which is accurate for $\sqrt{\lambda} r \ll 1$, was used to evaluate the correction.

Next it is verified to reproduce the correct RS limit in the case of $\lambda=0$ [6, 9, 12]. Then the case of $5 \mathrm{~d}$ Minkowski was examined and it was shown that the potential expresses the exact $5 \mathrm{~d}$ limit at any $r$. The similar situation was seen also in the $\mathrm{dS}$ bulk. Namely we can not see the region of $L \ll r$ so that the available region is restricted to the short range region. Furthermore, we could see the potential $U_{0} \sim 1 / r$ is not leading term but the "correction" $\Delta U \sim 1 / r^{2}$ is the dominant part. This indicates that the present universe is not embedded in the Minkowski or dS bulk when we consider according to our brane model.

As for $\mathrm{AdS}_{5}$ bulk, it would be possible to find the similar potential at large $r$ to the one of RS case when the parameters $\lambda$ and $\Lambda$ are appropriately chosen. However the essential difference lies in the fact that it would be expected that the ratio $\Delta U / U_{0}$ shifts from the RS case, $\Delta U / U_{0}=L^{2} /\left(2 r^{2}\right)$, and can be written as $\Delta U / U_{0}=f(L, \lambda) /\left(2 r^{2}\right)$. To study the meaning of this difference is an interesting problem from the theoretical 
viewpoint of AdS/CFT correspondence. We will discuss this issue in the future paper.

In conclusion, we can say that the favorable bulk configuration of the brane-world would be the $\mathrm{AdS}_{5}$ at the present universe. The formula (26) valid at $\sqrt{\lambda} r \ll 1$ is useful in comparing this theory with the measured corrections to Newton's law, because all the measurements are performed in the region $\sqrt{\lambda} r \ll 1$.

\section{Acknowledgments}

This work has been supported in part by the Grants-in-Aid for Scientific Research (13135223, 14540271) of the Ministry of Education, Science, Sports, and Culture of Japan.

\section{References}

[1] L. Randall and R. Sundrum, Phys. Rev. Lett. 83 (1999) 3370, (hep-ph/9905221).

[2] L. Randall and R. Sundrum, Phys. Rev. Lett. 83 (1999) 4690, (hep-th/9906064).

[3] I. Brevik, K. Ghoroku, S. D. Odintsov and M. Yahiro, Phys. Rev. 66 (2002) 064016, (hep-th/0204066).

[4] B. Bajc and G. Gabadadze, Phys. Lett. B474 (2000) 282, (hep-th/9912232). S. Nojiri and S.D. Odintsov, JHEP 0112 (2001) 033, (hep-th/0107134). M. Ito, (hep-th/0204113). P. Singh and N. Dadhich, (hep-th/0208080).

[5] S.B. Giddings, E. Katz and L. Randall, JHEP 03 (2000) 023, (hep-th/0002091).

[6] M.J. Duff and J.T. Liu, Phys. Rev. Lett. 85 (2000) 2052, (hep-th/0003237).

[7] N. Arkani-Hamed, S. Dimopoulos, G. Dvali and N. Kaloper, Phys. Rev. Lett. 84 (2000) 586 hep-th/9907209

[8] D.J. Chung and L. Everett, Phys. Rev. D64 (2001) 065022 hep-ph/0010103

[9] M. Ito, Phys. Lett. B 528 (2002) 269, (hep-th/0112224).

[10] A. Kehagias and K. Tamvakis, Class. Quant. Grav. 19 (2002) L185, (hep-th/0205009).

[11] The solutions $\psi$ given by Eq. (25) in [10] do not satisfy the boundary condition, $\psi^{\prime}\left(0_{+}\right) / \psi(0)=-\sigma /\left(12 M^{3}\right)$, required from (24) in [10]. If the $\psi$ are inserted on the LHS of the condition, it changes with the mass $m$ of KK modes, while the RHS does not. Thus, the $\psi$ have a wrong $m$ depencence. The normalization for $\psi$ is also obscure in [10]. The normalization factor depends in general on $m$. Then 
$\psi$ used in their analyses should change the $r$-dependence of the potential. These points would be essential in deriving the correction for $V(r)$.

[12] S. Nojiri and S.D. Odintsov, Phys. Lett. B548 (2002) 215, (hep-th/0209066).

[13] E. Kiritsis, N. Tetradis and T.N. Tomaras, (hep-th/0202037).

[14] N. Kaloper, Phys. Rev. D60 (1999) 123506, (hep-th/9905210).

[15] P. Binétruy, C. Deffayet, U. Ellwanger and D. Langlois, Phys. Lett. B477 (2000), (hep-th/9910219).

[16] N. Kaloper and A. Linde, Phys. Rev. D59 (1999) 101303, (hep-th/9811141). 